\title{
A Forward Regridding Method With Minimal Oversampling for Accurate and Efficient Iterative Tomographic Algorithms
}

\author{
Filippo Arcadu, Member, IEEE, Masih Nilchian, Member, IEEE, Alain Studer, \\ Marco Stampanoni, Member, IEEE, and Federica Marone
}

\begin{abstract}
Reconstruction of underconstrained tomographic data sets remains a major challenge. Standard analytical techniques frequently lead to unsatisfactory results due to insufficient information. Several iterative algorithms, which can easily integrate a priori knowledge, have been developed to tackle this problem during the last few decades. Most of these iterative algorithms are based on an implementation of the Radon transform that acts as forward projector. This operator and its adjoint, the backprojector, are typically called few times per iteration and represent the computational bottleneck of the reconstruction process. Here, we present a Fourier-based forward projector, founded on the regridding method with minimal oversampling. We show that this implementation of the Radon transform significantly outperforms in efficiency other state-of-the-art operators with $O\left(N^{2} \log _{2} N\right)$ complexity. Despite its reduced computational cost, this regridding method provides comparable accuracy to more sophisticated projectors and can, therefore, be exploited in iterative algorithms to substantially decrease the time required for the reconstruction of underconstrained tomographic data sets without loss in the quality of the results.
\end{abstract}

Index Terms-Tomography, iterative reconstruction algorithms, Radon transform, forward and backprojector, regridding method, minimal oversampling, kaiser-bessel, prolate-spheroidal wavefunctions.

\section{INTRODUCTION}

$\mathbf{T}$ HE word tomography refers to the method of reconstructing virtual cross-sections of an object using its projections acquired at different angles. The filtered backprojection (FBP)

Manuscript received March 12, 2015; revised August 17, 2015 and November 2, 2015; accepted January 5, 2015. Date of publication January 18, 2016; date of current version January 26, 2016. This work was supported by the Swiss National Science Foundation under Grant CR23I2-135550. The associate editor coordinating the review of this manuscript and approving it for publication was Prof. Jong Chul Ye.

F. Arcadu is with the Institute for Biomedical Engineering, Eidgenössische Technische Hochschule Zürich, Zürich 8092, Switzerland, and also with the Paul Scherrer Institute, Villigen 5232, Switzerland (e-mail: filippo.arcadu@psi.ch).

M. Nilchian is with the Biomedical Imaging Group, École Polytechnique Fédérale de Lausanne, Lausanne 1015, Switzerland (e-mail: masih.nilchian@ epfl.ch).

A. Studer is with the AIT Group, Paul Scherrer Institute, Villigen 5232, Switzerland (e-mail: alain.studer@psi.ch).

M. Stampanoni is with the Institute for Biomedical Engineering, Eidgenössische Technische Hochschule Zürich, Zürich 8092, Switzerland, and also with the SLS X-ray Tomography Group, Paul Scherrer Institute, Villigen 5232, Switzerland (e-mail: marco.stampanoni@psi.ch).

F. Marone is with the Paul Scherrer Institute, Villigen 5232, Switzerland (e-mail: federica.marone@psi.ch).

Color versions of one or more of the figures in this paper are available online at http://ieeexplore.ieee.org.

Digital Object Identifier 10.1109/TIP.2016.2516945 algorithm, introduced in the 70's [1], [2], is an analytical method, that still represents the standard tomographic reconstruction technique. It offers a good tradeoff between computational efficiency and accuracy. FBP provides high quality reconstructions, when a sufficient amount of projections is acquired and the noise affecting the experimental data is low with respect to the object signal [3].

In many tomographic applications, one or both of these conditions are not met due to experimental constraints. This occurs, in particular, when the total scan time needs to stay under a certain threshold due to dose limitations or to the evolution speed of the sample.

Many iterative algorithms have been designed to outperform FBP in the reconstruction of underconstrained datasets. These methods can easily incorporate a priori knowledge about the object or the image formation process and they usually provide better reconstructions than FBP, but at a much higher computational cost. Most iterative reconstruction algorithms are based on a forward projector and its adjoint (the backprojector), typically called few times per iteration. The forward projector usually corresponds to a discrete implementation of the Radon transform on a specific basis. The efficiency of this operator strongly determines the performance of the entire iterative procedure.

Standard tomographic forward projectors require $O\left(N^{3}\right)$ operations for an image of $N \times N$ pixels [4]-[12] and their computational cost remains substantial, even for highly optimized implementations on graphic processor units (GPUs) [13]. Recently, algorithms with $O\left(N^{2} \log _{2} N\right)$ complexity have been proposed to improve computational efficiency. These algorithms are based on three different strategies. The first group of projectors exploits the Fourier slice theorem [3]. Samples in the Fourier space are directly interpolated from a Cartesian to a polar grid [14]. These methods are characterized by poor accuracy and were never considered for iterative applications. The second category uses a hierarchical decomposition of either the line integrals [15]-[20] or the image domain [21]-[23]. In particular, the backprojector of [22] and [23] has shown great performance in terms of accuracy and speed. This latter work is, however, focused on analytical problems and an application to iterative algorithms has never been presented. The third class is based on the non-uniform fast Fourier transform with min-max interpolation [24]. This accurate and fast method has been exploited for iterative reconstruction techniques [25]. 
In this paper, we present a novel cost-effective application of the regridding method as tomographic forward projector with $O\left(N^{2} \log _{2} N\right)$ complexity. This method is compared for the first time with multiple state-of-the art implementations of the Radon transform. The role of the new forward projector in iterative procedures and, in particular, its influence on the quality of the results is also studied.

The regridding method, originally devised for image reconstruction in radio astronomy [26], [27], has been applied to a broad range of reconstructive imaging methods like magnetic resonance imaging (MRI) [28], [29], [33] and as backprojector in computer tomography (CT) [30], [32], [34], [35]. This method is designed to retrieve a $2 \mathrm{D}$ or a $3 \mathrm{D}$ signal from samples of its Fourier transform located on a nonCartesian lattice: the Fourier samples are, first, convolved with a window function onto a Cartesian grid, the IFFT is, then, applied and the signal in the real space is, finally, corrected from the windowing contribution. The speed and accuracy of the method depend entirely on the choice of the convolving window. There are, in particular, three milestones in the rather extensive literature about the regridding method: the prolate-spheroidal wavefunctions (PSWFs) and the kaiserbessels (KBs) are recognized as high performing convolving windows [30]; an oversampling factor of 2 , defined as ratio between the number of Fourier samples convolved onto the Cartesian grid and the number of pixels to be retrieved in the image space, is necessary for accurate reconstructions [31]; an efficient KB-based regridding method for MRI designed to work with oversampling $<2$ is introduced in [33].

Here the three previous results are integrated and an efficient forward projector tailored especially for iterative reconstruction algorithms is presented. The original contribution of this manuscript can be summarized as follows.

- Novel cost effective forward (and backward) regridding projector, based on the new combination of KB kernels and a minimal oversampling strategy for fast and accurate iterative reconstruction algorithms.

- Systematic comparison with multiple state-of-the-art implementations of the Radon transform, clearly demonstrating the advantages of the proposed projector for iterative reconstruction. Its inferior accuracy as standalone module is cancelled out rather than cumulated through iterations and its simplicity leads to significant performance (speed and memory) improvements of iterative techniques.

This paper is structured as follows. In Section II, the formulation and implementation details of the proposed forward regridding projector (FRP) with standard and minimal oversampling are presented. In Section III, the efficiency and accuracy of the FRP against state-of-the-art space- and Fourier-based forward operators are benchmarked. Section IV deals with the reconstruction of underconstrained simulated and experimental tomographic datasets with the separable paraboloidal surrogate (SPS) and the alternate direction method of multipliers (ADMM) implemented with the FRP and the most accurate among the projectors used in Section III. Section V offers a summary and final remarks.

\section{Proposed Method}

\section{A. Formulation}

This paper focuses on the tomographic reconstruction of a single 2D slice from line projections of an object in parallel beam geometry. However, Fourier methods have been previously applied also to fan-beam [36], [37] and cone-beam [38] geometry and the method proposed here can analogously be extended beyond this simple 2D case.

The set of line projections for one tomographic slice is called sinogram. The object to be reconstruncted is a finite integrable real function $f=f(x, y): \mathbb{R}^{2} \rightarrow \mathbb{R}$ with bounded support. The symbol ${ }^{\wedge}$ corresponds to the Fourier transform; $P_{\theta}(t)$ is the Radon transform of $f$ for an angle $\theta$ and a distance $t$ from the origin, as defined in [3]. Round and square brackets are used when referring respectively to continuous and discrete functions.

The Fourier slice theorem [3] states that:

$$
\hat{P}_{\theta}(\omega)=\hat{f}(\omega \cos \theta, \omega \sin \theta) .
$$

Thanks to (1), the problem of reconstructing $f$ from its projections $P_{\theta}(t)$ would be immediately solved, if the samples $\hat{f}\left[\omega_{i} \cos \theta_{j}, \omega_{i} \sin \theta_{j}\right]$ were located on a Cartesian grid. This is, however, not the case and the samples on the Cartesian grid need to be estimated from those available on a polar grid. Simple interpolation schemes (nearest neighbor, linear or bilinear interpolation), used for this approximation, lead to inaccurate reconstructions, since the localized interpolation error in Fourier space smears back onto the entire image, once the IFFT is computed.

In the regridding method (RM) [28], [31], the interpolation from the $\left[\omega_{i}, \theta_{j}\right]$ to the $\left[u_{m}, v_{n}\right]$ grid is, instead, performed as a convolution with a smooth window function $\hat{h}$, yielding more accurate results, depending on the chosen kernel. The standard RM for analytical CT reconstruction consists of five steps [30], [32], [35]:

1) FFT-1D of each projection $\longleftarrow \hat{P}_{\theta}$;

2) ramp filtering of the projections $\longleftarrow \hat{P}_{\theta}^{(f)}$;

3) convolution with window function $\longleftarrow \hat{f}^{(m)}=\hat{h} * \hat{P}_{\theta}^{(f)}$;

4) IFFT-2D of $\hat{f}^{(m)} \longleftarrow f^{(m)}$;

5) removal of the window function $\longleftarrow f=f^{(m)} / h$.

The last step, known as deapodization, is necessary, because computing $\hat{h} * \hat{f}$ in Fourier space is equivalent to calculating $h \cdot f$ in real space, so that removal of the convolving function is required if $f$ is sought. Accordingly, the forward regridding projector (FRP) is defined by the same steps listed above (ramp filtering excluded) in the reverse order:

1) pre-deapodization $\longleftarrow f^{(m)}=f / h$;

2) FFT-2D of $f^{(m)} \longleftarrow \hat{f}^{(m)}$;

3) convolution with window function $\longrightarrow \hat{P}_{\theta}=\hat{h} * \hat{f}^{(m)}$;

4) IFFT-1D of each polar slice of $\hat{P}_{\theta} \longleftarrow P_{\theta}$.

The FRP does not require density compensation factors, because, differently from the regridding method used for backprojection, the input samples are uniformly distributed in Fourier space. To simplify the algorithm, the convolving function $\hat{h}=\hat{h}(u, v)$ is chosen to be separable in $u, v$, implying that also $h=h(x, y)$ will be separable in $x, y$ :

$$
\hat{h}=\hat{h}(u, v)=\hat{\psi}(u) \cdot \hat{\psi}(v) \Longrightarrow h(x, y)=\psi(x) \cdot \psi(y)
$$


The FRP is defined as:

$$
\begin{aligned}
& P_{\theta_{i}}\left[t_{l}\right]=\mathcal{F}_{\omega}^{-1}\left\{\hat{P}_{\theta_{i}}\left[\omega_{j}\right]\right\}_{l}=\mathcal{F}_{\omega}^{-1}\left\{\left(\hat{h} * \hat{f}^{(m)}\right)\left[\omega_{j}, \theta_{i}\right]\right\}_{l} \\
&=\mathcal{F}_{\omega}^{-1}\left\{\sum_{\substack{u_{n} \in U_{i j} \\
v_{m} \in V_{i j}}}^{\hat{\psi}\left[g_{1}\left(\omega_{j, u}-u_{n}\right)\right] \hat{\psi}\left[g_{1}\left(\omega_{j, v}-v_{m}\right)\right]} \hat{C}_{i, j, n, m}\right. \\
&\left.\times \hat{f}^{(m)}\left[u_{n}, v_{m}\right]\right\} \\
&=\mathcal{F}_{\omega}^{-1}\left\{\sum_{\substack{u_{n} \in U_{i j} \\
v_{m} \in V_{i j}}} C_{i, j, n, m} \mathcal{F}_{x, y}\left\{\frac{f\left[x_{p}, y_{q}\right]}{\psi\left[g_{2} x_{p}\right] \psi\left[g_{2} y_{q}\right]}\right\}_{u_{n}, v_{m}}\right\}_{l}
\end{aligned}
$$

where $\mathcal{F}_{a}$ is the (discrete) Fourier transform with respect to the variable $a,\{\ldots\}$ indicates a collection of discrete values, $\omega_{j, u}=\omega_{j} \cos \theta_{i}, \omega_{j, v}=\omega_{j} \sin \theta_{i}, U_{i j}$ and $V_{i j}$ are respectively the sets of values of $u_{n}$ and $v_{m}$ inside the interpolation support, $g_{1}$ and $g_{2}$ are factors to map distances in the polar and Cartesian grid into look-up tables (LUTs) indices.

The accuracy and efficiency of the RM (and, therefore, of the FRP) lie entirely in the choice of the convolving function $\hat{h}$. In terms of accuracy, the optimal kernel is an infinite sinc function [30], which is, however, computationally impractical. For finite kernels and, therefore, superior efficiency, the smaller the supports of $h$ and $\hat{h}$ are, the better the performance of the method [30]. If $h$ has compact support in real space, the interpolation error is still smeared out on the whole image, but it can be expected to remain as local as possible and the reconstruction will be accurate [31]; if $\hat{h}$ has compact support in Fourier space, only few datapoints in the support region will contribute to the convolution and the method will be computationally efficient. The accuracy of a convolution kernel $\hat{h}$ is strongly determined by the shape (in particular, the rolloff) of the central lobe and the amplitude of the aliasing sidelobes characterizing $h$. The deapodization removes the rolloff induced by this central lobe, especially at the borders of the output image, but, at the same time, it amplifies the aliasing sidelobes [31]. Aliasing contamination from sidelobes can be reduced by oversampling [30], [31], i.e. artificially extending the image field-of-view (FOV) and ignoring the outer portion of the image after reconstruction. In this way, smaller sidelobes will alias back into a wider central lobe, whose tails are neglected, once the output array is cropped. The extended central lobe also tapers less steeply, so that less rolloff correction is required. For a more detailed discussion on the optimal characteristics of convolution kernels, the reader is referred to the abovementioned references.

An oversampling $\alpha=G / N=2.0$, with $G$ the size of the grid, where the interpolation takes place, and $N$ the size of the original input grid, guarantees accurate reconstructions with the RM [30], [31]. The aliasing error is a metric to compare the performance of different convolving functions and has the following pixel-based form [31], [33]:

$$
\epsilon(i)=\sqrt{\frac{\sum_{p \neq 0}[h(i+G p)]^{2}}{[h(i)]^{2}}} .
$$

\section{B. FRP With Prolate Spheroidal Wavefunctions}

As outlined above, the energy of the ideal convolution kernel should be maximally concentrated in a compact support both in real and Fourier space. We introduce the operators $P_{T}$ and $P_{\Omega}$, that create respectively time- and band-limited functions:

$$
\begin{aligned}
P_{T} f(t) & =\Theta_{T} f(t) \quad P_{\Omega} f(t)=\mathcal{F}_{\omega}^{-1}\left[\Theta_{\Omega} \mathcal{F}_{t}(f(t))\right] \\
\Theta_{T}(t) & =\left\{\begin{array}{ll}
1 & \text { if }|t| \leq T / 2 \\
0 & \text { if }|t|>T / 2
\end{array} \Theta_{\Omega}(\omega)= \begin{cases}1 & \text { if }|\omega| \leq \Omega / 2 \\
0 & \text { if }|\omega|>\Omega / 2\end{cases} \right.
\end{aligned}
$$

The prolate spheroidal wavefunctions (PSWFs) are the eigenfunctions of the self-adjoint operator $P_{\Omega} P_{T}$ [39]:

$$
P_{\Omega} P_{T} \psi_{i}(t)=\int_{-T / 2}^{T / 2} d t^{\prime} \frac{\sin \left(\Omega\left(t-t^{\prime}\right)\right)}{\pi\left(t-t^{\prime}\right)} \psi_{i}\left(t^{\prime}\right)=\lambda_{i} \psi_{i}(t) .
$$

If $\psi_{i}$ 's are bandlimited, the PSWF of 0 -th order, $\psi_{0}$, with the largest eigenvalue $\lambda_{0}$, has the highest amount of energy $E_{0}=\left\|P_{T} \psi_{0}\right\|^{2}$ in the interval $[-T / 2, T / 2]$ [39]. From the eigenvalue equation (6), no analytical expression for the PSWFs can be obtained; however, several approximations exist, depending on the magnitude of the parameter $c=$ $T \Omega / 2$ [39]. Since $T$ should not be larger than \pm 1 to guarantee an accurate deapodization correction [35], PSWFs are best approximated using spherical Bessel functions, expressed as normalized linear combinations of even Legendre polynomials in Fourier space [39]:

$$
\hat{\psi}_{0}^{(a)}(k)=\frac{\sum_{n=0}^{N_{a}} c_{2 n} L_{2 n}(k)}{\sum_{n=0}^{N_{a}} c_{2 n} L_{2 n}(0)},
$$

where the superscript ${ }^{(a)}$ stands for "approximated", $L_{n}$ is the Legendre polynomial of degree $n$ and $\left\{c_{2 n}\right\}$ are the expansion coefficients. The inverse Fourier transform of a series of even Legendre polynomials corresponds to a series of even spherical Bessel functions, that can themselves be expanded in the interval $[-1,1]$ in terms of even Legendre polynomials [40], [41]. According to this argument, $\psi_{0}^{(a)}(x)$ represents a scaled version of $\hat{\psi}_{0}^{(a)}(k)$ :

$$
\psi_{0}^{(a)}(x)=C \cdot \hat{\psi}_{0}^{(a)}\left(x \frac{S}{G}\right),
$$

where $S$ is the density of the convolution LUT and $C \in \mathbb{R}_{0}$ is a constant.

It has been shown that an analytical regridding algorithm, based on this approximation of the PSWFs of 0-th order, is about 20 times faster than FBP, without accuracy degradations [35]. The FRP has been implemented analogously with 
separable convolving kernel and deapodizer given respectively by (7) and (8). The following additional parameters were used: $\alpha=2.0, N_{a}=10$ and $S=2048$. This implementation is abbreviated with FRP-PSWF. Since $\alpha=2$ guarantees accurate results [31], we consider this oversampling as "standard".

\section{FRP With Minimal Oversampling}

Due to the lack of an analytical expression for the PSWFs, it is difficult, in this case, to optimize the oversampling and other parameters as a function of the aliasing error (4). Kaiser Bessels (KBs) kernels have shown great accuracy compared to other convolving windows like two- and three-term cosine, Gaussian and B-splines [31], although their aliasing error is slightly higher than for the PSWFs approximation in (7). The simple analytical expression of KBs kernels enables, however, an optimization of their shape as a function of the oversampling [33]. The formulas for the KB window and its inverse Fourier transform are [42]:

$$
\begin{aligned}
\hat{h}\left(k_{x}\right) & =\frac{G}{W} I_{0}\left(\beta \sqrt{1-\left(\frac{2 G k_{x}}{W}\right)^{2}}\right) \text { for }\left|k_{x}\right| \leq \frac{W}{2 G}, \\
h(x) & =\frac{\sin \sqrt{\left(\frac{\pi W x}{G}\right)^{2}-\beta^{2}}}{\sqrt{\left(\frac{\pi W x}{G}\right)^{2}-\beta^{2}}},
\end{aligned}
$$

where $I_{0}$ is the zero-th modified Bessel function, $W$ is the size of the convolving kernel and $\beta$ is the tapering parameter, that determines how fast the KB drops to zero. $\alpha<2$ yields a substantial decrease in run time and memory required for the reconstruction compared to the case of the RM with standard oversampling. The shape optimization of the KB kernel as a function of oversampling gives the following results [33]:

$$
\begin{aligned}
& \beta=\pi \sqrt{\left(\frac{W}{\alpha}\right)^{2} \cdot\left(\alpha-\frac{1}{2}\right)^{2}-0.8} \\
& S=\frac{0.91}{\alpha \cdot \epsilon_{s}} \text { for NN } S=\frac{1}{\alpha} \sqrt{\frac{0.37}{\epsilon_{s}}} \text { for LIN, }
\end{aligned}
$$

where NN and LIN refer, respectively, to the nearest neighbor and linear interpolation scheme to sample the convolution LUT and $\epsilon_{s}$ is the maximum allowed sampling error. The FRP with minimal oversampling has been implemented with a KB kernel based on (11) and (12), setting $\epsilon_{s}=0.01, \alpha=1.125$ and choosing a linear interpolation scheme for the LUT. The FRP with these settings, abbreviated as FRP-KB, computes artefact-free forward projections in the shortest time and with the smallest memory allocation.

\section{Further Details}

The kernel width is set to $W=14.0 / \pi$ for both FRP-PSWF and FRP-KB. Experiments with the filtered adjoint operators, conducted on the Shepp-Logan (SL) phantom [43], show that for $W \in[18 / \pi, 20 / \pi]$ the reconstruction is affected by a "blackening" artefact at the sides of the image, because the intepolation is not local anymore, whereas for $W \in[4 / \pi, 10 / \pi]$ the interpolation support is too small and the reconstruction is consequently affected by bright symmetric curves. Reconstructions look reasonable, by visual inspection, for $W \in(10 / \pi, 18 / \pi)$; in our implementation, $W=14.0 / \pi$ provides the most accurate results.

Moreover, we recall that the backprojector is the adjoint (not the inverse!) operator of a forward projector. From a computational point of view, the backprojector performs all operations of the forward projector, but in reverse order. Density compensation factors (DCFs) are not needed for the backprojector, when coupled to the forward operator inside an iterative scheme. The DCFs come into play only when the backprojector is used for analytical tomographic reconstruction. In such case, they correspond to a discrete version of the ramp filter, analogously to FBP.

The FRP operators are implemented in $\mathrm{C}$ with the fast FFTW library [44] and the pseudocode is shown in Appendix. The 1D functions $\hat{\psi}$ and $\psi$ are stored in precomputed LUTs to allow for fast computations.

\section{E. Algorithm Complexity}

The cost of the FRP lies in the convolution and the call of FFT/IFFT. Given an input image of $N \times N$ pixels, an oversampling ratio $\alpha$, a kernel width $W$ and a number of views $M$, the convolution amounts to approximately $W M N$ operations, whereas the overall call of FFT/IFFT corresponds to $\alpha^{2} N^{2} \log _{2}(\alpha N)+\alpha M N \log _{2}(\alpha N)$ (1-time FFT-2D and $M$-times IFFT-1D) floating operations, that represents the leading cost term when $N$ is big enough.

\section{ACCURACY ASSESSMENT}

\section{A. Benchmark Procedure}

The accuracy of the proposed forward projectors has been assessed with a phantom for which the analytical Radon transform can be computed. The SL phantom [43] consists of 10 roto-translated ellipses and, is, therefore, suited for this task [3]. We use here a modifed version of the original SL featuring higher contrast to improve visual perception.

The FRP-PSWF and FRP-KB are benchmarked against multiple state-of-the-art implementations of the Radon transform, listed in the following.

The ray-driven (RT-RAY) [9] and distance-driven (RT-DIST) [10] forward projectors are widely used and are characterized by $O\left(N^{3}\right)$ complexity.

The Radon transform based on a cubic B-spline basis (RT-BSPLINE) [12] is a very accurate projector, superior, for instance, to implementations on pixel [8] and Kaiser-Bessel basis [11]. It is characterized by $O\left(N^{3}\right)$ complexity. For its implementation, a LUT of projected B-spline tensor products is precomputed for each view $(S=2048)$ and used inside a standard pixel-based forward projector.

The hierarchical filtered backprojection [22] treats the reconstruction of an $N \times N$ image as the sum of shifted reconstructions of smaller images centered at the origin. While the image grid is recursively halved, the input sinogram is halved in angles and pixels, since each subimage 
requires fewer projections for the reconstruction. Thanks to this decomposition scheme, the backprojection is performed with $O\left(N^{2} \log _{2} N\right)$ operations. We used the implementation described in [22] and [23] to build the corresponding forward projector (FHFP). The parameters ruling the image/sinogram decomposition and the choice of the interpolation schemes can strongly influence the accuracy and efficiency of this projector. Using the performance study in [23] as guide, we selected the best parameters for accurate results, when studying the operator accuracy. For the computational cost evaluation, parameters providing fastest artefact-free (at visual inspection) forward/backprojection have instead been used.

The non-uniform fast Fourier transform allows to Fourier transform finite-length signals to a non-uniformly sampled frequency space. Similarly to the regridding method, it can be used to reconstruct a signal from a non-Cartesian collection of samples in Fourier space. Several versions exist ([45]-[48]): the non-uniform fast Fourier transform using min-max interpolation represents, so far, the most accurate implementation of this method [24]. The forward projector based on this technique (NUFFT) [25], [37] requires $O\left(N^{2} \log _{2} N\right)$ operations, but also a significant amount of additional calculations and memory to precompute and store shift-variant interpolators. If NUFFT makes use of a shift-invariant kernel, the technique does not differ from a standard regridding method. The performance of NUFFT depends on several parameters like the type of kernel, size of the convolution window, sampling density of the LUT and oversampling ratio. In the comparison of the forward projector, NUFFT is used with the setting providing the best possible accuracy, according to the study presented in [25]. This setting implies a shift-variant kernel of KaiserBessel, optimized on the basis of the min-max principle, $\alpha=2.0$ and $W=7$.

\section{B. Analysis Tools}

The accuracy of sinograms and reconstructed images obtained with different forward projectors is assessed using the following standard metrics: root mean square error (RMSE), peak-signal-to-noise-ratio (PSNR) [49] and mean structural similarity index (MSSIM) [50]. For the accuracy assessment of the projectors in iterative schemes (Section IV-C), a linearly regressed version $\hat{I}_{\text {regr }}$ of the reconstructed slice $(I)$ is used for the metrics computation. In this way, the obtained score is less biased by the different gray scales, characterizing $I$ and the reference image, $O$, guaranteeing fair comparisons. Reconstruction methods exploiting total variation can, in fact, lead to slight shrinkage of the sparse coefficients [51]. $\hat{I}_{\text {regr }}$ is computed in the following way:

$$
\hat{I}_{\text {regr }}=\underset{I_{\text {regr }}}{\operatorname{argmin}}\left\|I_{\text {regr }}-O\right\|_{2}^{2} \text { s.t. } I_{\text {regr }}=a \cdot I+b
$$

where $a, b \in \mathbb{R}$.

\section{Comparison of Forward Projectors}

The accuracy assessment of the two proposed forward projectors (FRP-PSWF, FRP-KB) is assessed against the
TABLE I

RMSE, PSNR AND MSSIM VALUES FOR SL SINOGRAMS WITH 805 Views $\times 512$ PiXels Generated By the AlgorithmS

LISTED IN THE FIRST COLUMN. THE REFERENCE SINOGRAM IS REPRESENTED BY THE ANALYTICAL RADON TRANSFORM OF SL

\begin{tabular}{rrrr}
\hline & RMSE & PSNR & MSSIM \\
\hline FRP-PSWF & 4.14 & 43.99 & 0.82 \\
\hline FRP-KB & 4.25 & 42.75 & 0.80 \\
\hline RT-RAY & 3.34 & 44.84 & 0.90 \\
\hline RT-DIST & 3.34 & 44.85 & 0.91 \\
\hline FHFP & 3.51 & 44.42 & 0.88 \\
\hline NUFFT & 3.28 & 45.01 & 0.90 \\
\hline RT-BSPLINE & 1.24 & 53.44 & 0.89 \\
\hline
\end{tabular}

five operators introduced in III-A (RT-RAY, RT-DIST, FHFP, NUFFT, RT-BSPLINE).

The results of this section are focused on the forward projection and subsequent analytical reconstruction of a SL phantom with $512 \times 512$ pixels. These experiments were also performed on SL phantoms with other resolutions $(128 \times 128,256 \times 256$, $1024 \times 1024,2048 \times 2048)$ and different number of views. The results show that the observed trends are independent from the phantom size and the angular sampling.

Sinograms with 805 views $\times 512$ pixels have been computed using the seven above-mentioned forward projectors and are compared with the analytical Radon transform of the SL phantom by means of the RMSE, PSNR and MSSIM values (Tab. I). The figures of merit clearly show that the FRPs perform the worst, while RT-BSPLINE provides the most accurate sinogram. The other operators (NUFFT, RT-RAY, RT-DIST and FHFP) are characterized by an intermediate accuracy. The same accuracy trends are observed for the backprojectors, when used as standalone modules for the reconstruction (with a ramp filter) of the analytical SL sinogram. The filtered adjoint RT-BSPLINE provided the most accurate reconstruction, followed by the filtered adjoint NUFFT, RT-RAY, RT-DIST, FHFP, FRP-PSWF and, finally, FRP-KB.

In a second experiment, the effect of coupling the filtered adjoint operator to the corresponding forward projector is studied in relation to the accuracy of the forward projector itself. Each sinogram is filtered with a standard ramp kernel in Fourier space and reconstructed using the corresponding backprojector. The chosen number of views $805 \simeq 512 * \pi / 2$ guarantees that these analytical reconstructions are not affected by aliasing artefacts [3], ensuring that we are really quantifying the accuracy of the entire projection-reconstruction process for each operator. The results in Tab. II show metric scores very close to each other, contrary to the trend seen in Tab. I. RT-BSPLINE confirms its highest accuracy, but NUFFT, RT-RAY and RT-DIST perform in a very similar way to the FRP operators. In this case, FHFP shows the lowest accuracy. This second experiment indicates that either the coupling 
TABLE II

RMSE, PSNR AND MSSIM VALUES FOR THE RECONSTRUCTIONS OF THE SL SiNOGRAMS WiTH 805 VIEWS $\times 512$ PIXELS GENERATED B Y THE ALGORITHMS LISTED IN THE First Column. The Recostructions Were PERFORMED By MEANS OF THE RAMP-FILTERED ADJOINT OPERATOR CORRESPONDING TO EACH SiNOGRAM. THE REFERENCE IMAGE IS THE ORIGINAL SL PHANTOM. THE SCORES WERE COMPUTED INSIDE THE RECONSTRUCTION CIRCLE

\begin{tabular}{rrrr}
\hline & RMSE & PSNR & MSSIM \\
\hline FRP-PSWF & 0.09 & 26.00 & 0.24 \\
\hline FRP-KB & 0.10 & 25.90 & 0.21 \\
\hline RT-RAY & 0.10 & 25.80 & 0.23 \\
\hline RT-DIST & 0.10 & 25.80 & 0.24 \\
\hline FHFP & 0.11 & 24.95 & 0.13 \\
\hline NUFFT & 0.11 & 25.45 & 0.24 \\
\hline RT-BSPLINE & 0.09 & 27.42 & 0.37 \\
\hline
\end{tabular}

TABLE III

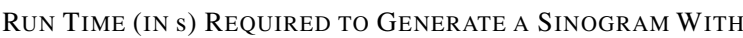
2048 PIXELS AND 800, 1600 AND 3200 VIEWS FOR THE Algorithms Listed IN the First Column. The USED HARDWARE IS AN INTEL(R) CORE(TM) i7 $-3520 \mathrm{M} \mathrm{CPU} 2.90 \mathrm{GHz}$

TIME (s)

\begin{tabular}{rrcr}
\hline & 800 views & 1600 views & 3200 views \\
\hline FRP-PSWF & 3.22 & 4.60 & 5.13 \\
\hline FRP-KB & 0.68 & 0.94 & 1.47 \\
\hline FHFP & 11.24 & 15.88 & 44.88 \\
\hline NUFFT & 7.83 & 10.12 & 16.01 \\
\hline RT-BSPLINE & 438.60 & 875.71 & 1775.04 \\
\hline
\end{tabular}

forward-adjoint operator and/or the effect of a simple ramp filter may reduce or even cancel the accuracy superiority of a well-performing projector with respect to others of inferior quality. Computed sinograms and reconstructed slices are not shown because differences are not detectable by visual inspection. The same trend was obtained when performing the ramp filtering through convolution in real space.

Tab.III and IV report the time needed and the memory allocated to create a SL sinogram with 2048 pixels and 800, 1600 and 3200 views. The used hardware is an Intel(R) Core(TM) i7-3520M CPU 2.90GHz. The FRP-KB, FRP-PSWF, FHFP and RT-BSPLINE operator were all implemented in C. For the NUFFT projector, the code available on [52] has been used, including the same FFTW library [44] as the FRP operators. Values in Tab.III include the time required to build the look-up-table, if required by the algorithm (all except FHFP). FRP-KB is, on average, faster by a factor of 4.4 , 11.0, 21.3 and 928 compared to FRP-PSWF, NUFFT, FHFP and RT-BSPLINE. The computational cost for all projectors,
TABLE IV

Memory (IN MB) Required to Generate a Sinogram With 2048 PIXELS AND RESPECTIVELY 800, 1600 AND 3200 VIEWS FOR THE ALGORITHMS LISTED IN THE FIRST COLUMN

MEMORY (MB)

\begin{tabular}{rrcr}
\hline & 800 views & 1600 views & 3200 views \\
\hline FRP-PSWF & 207.93 & 214.49 & 227.61 \\
\hline FRP-KB & 70.28 & 76.84 & 89.96 \\
\hline FHFP & 26.05 & 35.33 & 53.88 \\
\hline NUFFT & 234.13 & 266.91 & 332.46 \\
\hline RT-BSPLINE & 29.89 & 43.00 & 69.23 \\
\hline
\end{tabular}

except RT-BSPLINE, scales similarly with the number of views in particular for 800 and 1600 projections. Among the Fourier-based methods, FRP-KB allocates, on average, $64 \%$ and $70 \%$ less memory than FRP-PSWF and NUFFT, respectively. RT-BSPLINE requires, instead, more memory than a standard pixel-based forward projector because the precomputed LUT has to be finely sampled to guarantee high accuracy. RT-RAY and RT-DIST are not listed in these tables, since the implementations used for this work are based on GPUs. However, extrapolations suggest that a single core implementation of RT-DIST could require approximately the same memory, but larger computational cost than FHFP. On the other hand, RT-RAY may require more memory than RT-DIST and longer times than RT-BSPLINE.

\section{Application to Iterative RECONSTRUCTION AlgORITHMS}

In this section, the usage of the proposed forward projection operator inside iterative algorithms is investigated. The study is aimed at assessing experimentally the connection between the accuracy of the forward projectors and the quality of the iterative reconstruction. Two completely different iterative methods are used in the following: the separable paraboloidal surrogate and the alternate direction method of multipliers.

\section{A. Separable Paraboloidal Surrogate}

The separable paraboloidal surrogate (SPS) [53] is an algorithm for penalized likelihood based on Poisson statistics, modeling the measurements at the detector. The SPS globally converges to a unique minimizer, easily allows to enforce non-negativity constraints and to exploit non-quadratic convex penalty functions. SPS is implemented with the edgepreserving regularization introduced in [53] and [54]. For further details about this well-established iterative method for tomographic reconstruction, please refer to [53].

\section{B. Alternate Direction Method of Multipliers}

The alternate direction method of multipliers (ADMM) [55] is an iterative scheme suitable to minimize a large variety of functionals, including those with L1-norm terms. 
Thus, the ADMM can be used to solve a tomographic reconstruction problem with total variation regularization:

$$
\hat{\mathbf{x}}=\underset{\mathbf{x}}{\operatorname{argmin}}\left[\|\mathbf{A x}-\mathbf{b}\|_{2}^{2}+\lambda \cdot \mathrm{TV}_{\Omega}(\mathbf{x})\right] \text {. }
$$

$\mathbf{A}$ is the matrix representation of the forward projector, $\mathbf{b}$ is the sinogram, $\mathbf{x}$ is the unknown object and $\lambda \in \mathbb{R}$ determines the weight of the regularization; TV stands for total variation, defined as [56]:

$$
\operatorname{TV}_{\Omega}(f)=\int_{\Omega}|\nabla f(\mathbf{x})| d \mathbf{x}=\|\nabla f(\mathbf{x})\|_{1} .
$$

The problem (14) can be mapped into the minimization of the following augmented Lagrangian [57]:

$$
\begin{aligned}
\mathcal{L}_{\mu}(\mathbf{x}, \mathbf{u}, \boldsymbol{\alpha})= & \frac{1}{2}\|\mathbf{A} \mathbf{x}-\mathbf{b}\|_{2}^{2}+\lambda \sum_{\mathbf{k}}\left\|\mathbf{u}_{\mathbf{k}}\right\|_{1} \\
& +\boldsymbol{\alpha}^{T}(\mathbf{L} \mathbf{x}-\mathbf{u})+\frac{\mu}{2}\|\mathbf{L x}-\mathbf{u}\|_{2}^{2}
\end{aligned}
$$

where $\boldsymbol{\alpha}$ are the Lagrangian multipliers, $\mathbf{L}$ is the gradient operator and $\mathbf{u}$ is an auxiliary variable. The ADMM iteratively minimizes $\mathcal{L}_{\mu}(\mathbf{x}, \mathbf{u}, \boldsymbol{\alpha})$ by sequentially solving smaller problems; each iteration involves two sub-optimizations with respect to $\mathbf{x}$ and to $\mathbf{u}$, followed by the update of $\boldsymbol{\alpha}$ :

$$
\begin{aligned}
& \text { 1) } \mathbf{x}_{k+1} \longleftarrow \underset{\mathbf{x}}{\operatorname{argmin}} \mathcal{L}_{\mu}\left(\mathbf{x}, \mathbf{u}_{k}, \boldsymbol{\alpha}_{k}\right) \\
& \text { 2) } \mathbf{u}_{k+1} \longleftarrow \underset{\mathbf{u}}{\operatorname{argmin}} \mathcal{L}_{\mu}\left(\mathbf{x}_{k+1}, \mathbf{u}, \boldsymbol{\alpha}_{k}\right) \\
& \text { 3) } \boldsymbol{\alpha}_{k+1} \longleftarrow \boldsymbol{\alpha}_{k}+\mu\left(\mathbf{L} \mathbf{x}_{k+1}-\mathbf{u}_{k+1}\right) .
\end{aligned}
$$

In step 1), the conjugate gradient (CG) method [58] is applied to the following linear system:

$$
\left(\mathbf{A}^{\dagger} \mathbf{A}+\mu \mathbf{L}^{\dagger} \mathbf{L}\right) \mathbf{x}=\mathbf{A}^{\dagger} \mathbf{b}+\mu \mathbf{L}^{\dagger}\left(\mathbf{u}_{k}-\frac{\boldsymbol{\alpha}_{k}}{\mu}\right) .
$$

The subproblem of step 2) is solved through a shrinkage operation:

$$
\mathbf{u}_{k+1}=\max \left\{\left|\mathbf{L x}_{k+1}+\frac{\boldsymbol{\alpha}_{k}}{\mu}\right|-\frac{\lambda}{\mu}, 0\right\} \operatorname{sgn}\left(\mathbf{L} \mathbf{x}_{k+1}+\frac{\boldsymbol{\alpha}_{\boldsymbol{k}}}{\mu}\right) .
$$

Our implementation of the ADMM performs 4 of CG (17) loops for the $\mathbf{x}$-subproblem, whereas $\mu$ and $\lambda$ are set to 1.0. This setting provides accurate reconstructions when dealing with the group of simulated and real data used in the following Section. Neither trends regarding the accuracy nor the convergence of the ADMM implementations change, if a different number of CG iterations and/or different values for $\mu$ and $\lambda$ are used.

\section{Experiments}

SPS and ADMM have been implemented with the proposed projectors (SPS/ADMM-PSWF, SPS/ADMM-KB) and with RT-BSPLINE (SPS/ADMM-BSPLINE), since this latter operator has shown the best accuracy in the experiments presented in III-C.

The convergence of the iterative algorithms has been studied through the tomographic reconstruction of a SL sinogram with 200 views $\times 128$ pixels. In this experiment, the number

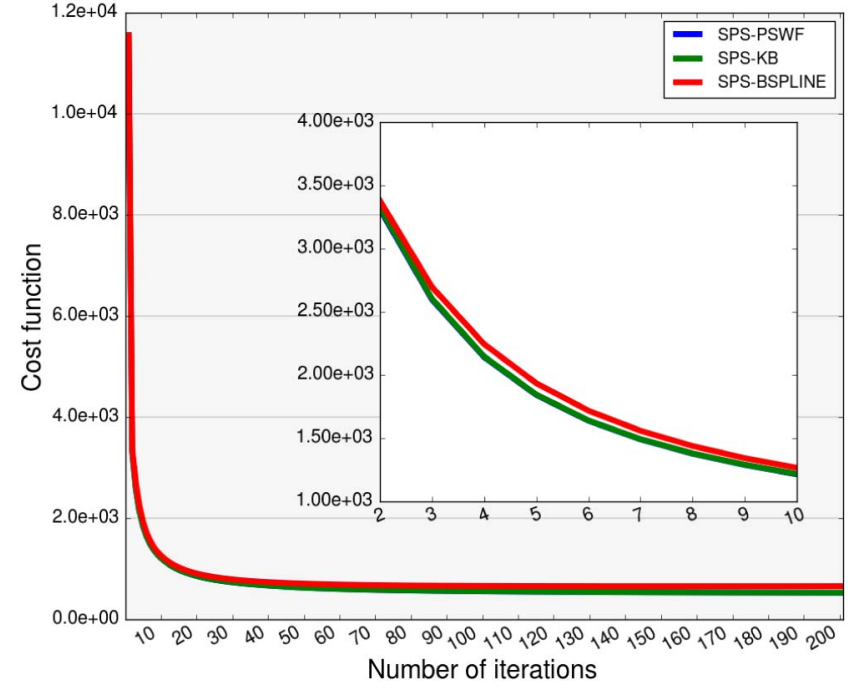

Fig. 1. Cost function of the SPS against the number of iterations for the three SPS implementations. The plot corresponds to the reconstruction of a noiseless SL sinogram with 128 views $\times 200$ pixels.

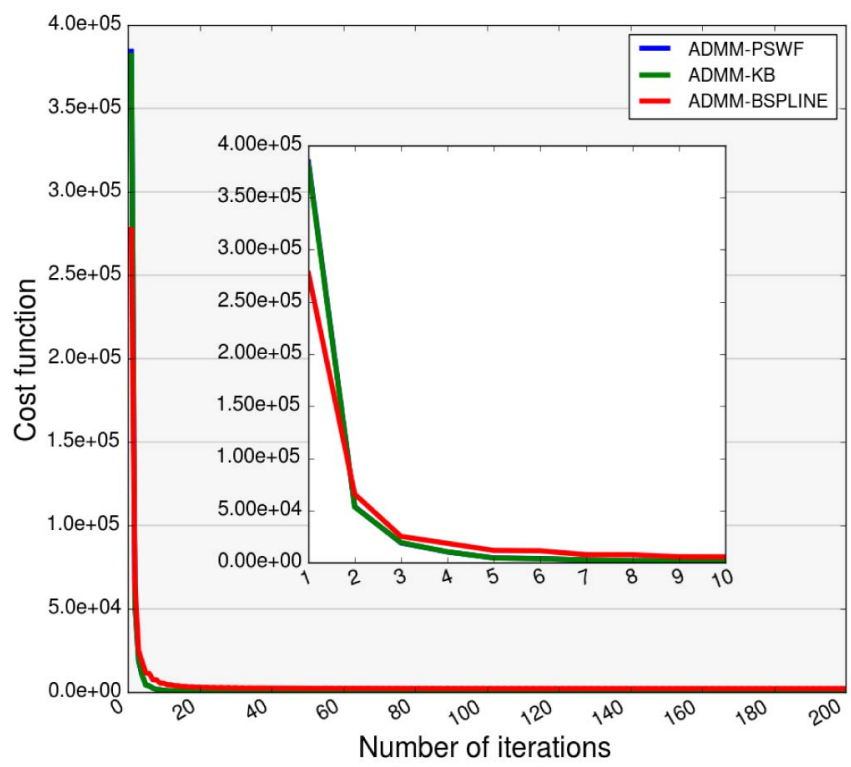

Fig. 2. Cost function of the ADMM (14) against the number of iterations for the three ADMM implementations. The plot corresponds to the reconstruction of a noiseless SL sinogram with 128 views $\times 200$ pixels.

of iterations was 200. The cost function plots in Fig. 1, 2 show that, for both SPS and ADMM, the convergence is not significantly affected by the choice of the forward operator. The three implementations of each iterative method show a slight mismatch only for the first 10 iterations (Fig. 1, 2). The following reconstructions of simulated and real data are computed by stopping the iterative algorithm when the $L_{2}$-norm of the relative difference between reconstructions of subsequent iterations is smaller than a threshold $\epsilon=0.01$, i.e., $\left\|\mathbf{x}_{k+1}-\mathbf{x}_{k}\right\|_{2}^{2} /\left\|\mathbf{x}_{k}\right\|_{2}^{2}<0.01$. Following this criterion, the iterative procedure was usually stopped after few tens of iterations. To check whether convergence was effectively achieved in this way, we computed the maximum difference 


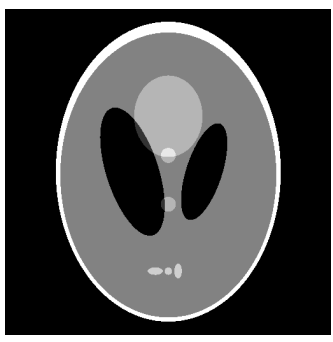

(a)

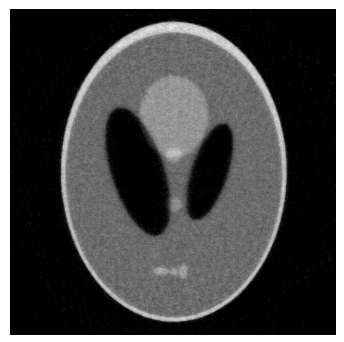

(b)

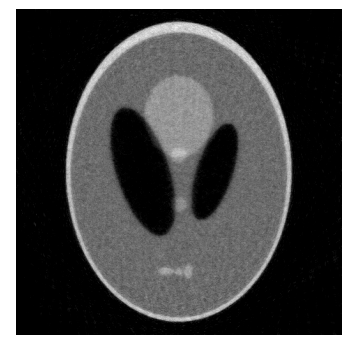

(c)

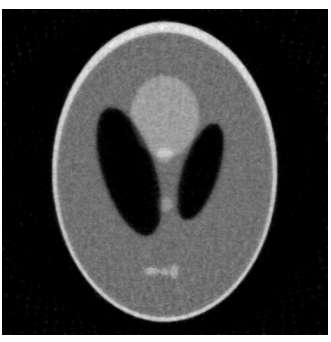

(d)

Fig. 3. SPS reconstructions of a SL sinogram with 50 views $\times 512$ pixels and additional Gaussian noise with $\sigma=2.4 \%$ of the sinogram mean. (a) Phantom. (b) Reconstr. with SPS-PSWF. (c) Reconstr. with SPS-KB. (d) Reconstr. with SPS-BSPLINE.

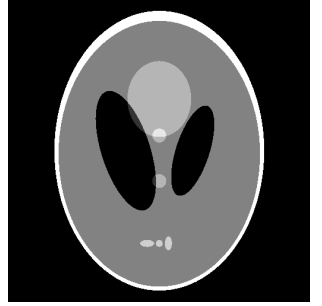

(a)

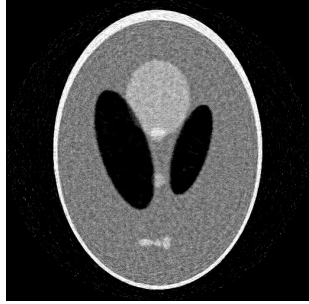

(b)

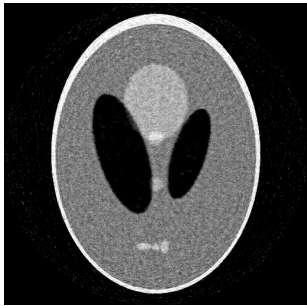

(c)

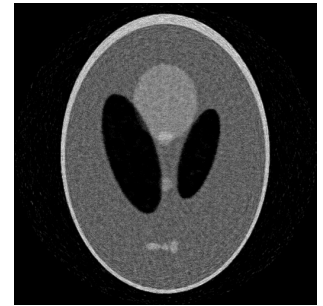

(d)

Fig. 4. ADMM reconstructions of a SL sinogram with 50 views $\times 512$ pixels and additional Gaussian noise with $\sigma=2.4 \%$ of the sinogram mean (a) Phantom. (b) Reconstr. with ADMM-PSWF. (c) Reconstr. with ADMM-KB. (d) Reconstr. with ADMM-BSPLINE.

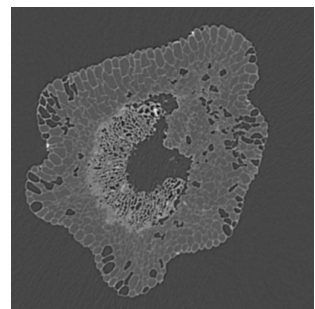

(a)

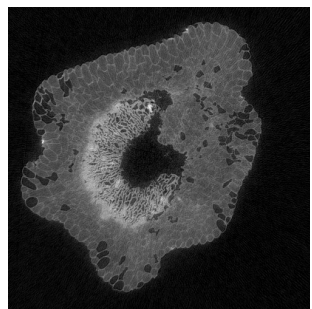

(b)

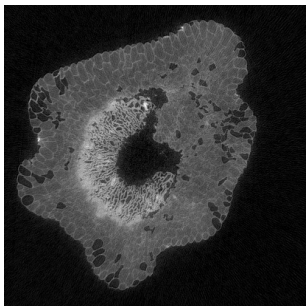

(c)

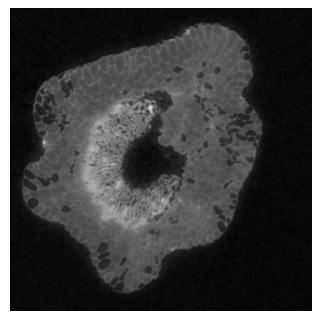

(d)

Fig. 5. SPS reconstructions of the sinogram (300 views $\times 2560$ pixels) of a modern seed specimen. FBP-FULL is the FBP reconstruction of the fully sampled sinogram with 1441 views $\times 2560$ pixels Sample courtesy: S. Smith, University of Michigan. (a) Reconstr. with FBP-FULL. (b) Reconstr. with SPS-PSWF. (c) Reconstr. with SPS-KB. (d) Reconstr. with SPS-BSPLINE.

TABLE V

RMSE, PSNR AND MSSIM VALUES FOR THE SPS RECONSTRUCTIONS OF THE SL SinOGRAM SHOWN IN Fig.3. THE SCORES ARE COMPUTED INSIDE THE RECONSTRUCTION CIRCLE

\begin{tabular}{rccr}
\hline & RMSE & PSNR & MSSIM \\
\hline SPS-PSWF & 0.14 & 22.86 & 0.11 \\
\hline SPS-KB & 0.14 & 22.86 & 0.11 \\
\hline SPS-BSPLINE & 0.16 & 21.67 & 0.12 \\
\hline
\end{tabular}

between the reconstruction obtained with the aforementioned criterion and the one computed after 200 iterations (procedure suggested in [25]): for both SPS and ADMM and for all projectors, the maximum difference was always under $2 \%$.

In the first experiment, an underconstrained SL sinogram with 50 views $\times 512$ pixels has been used. Gaussian noise with $\sigma=2.4 \%$ of the sinogram mean has been added.
TABLE VI

RMSE, PSNR AND MSSIM VALUES FOR THE ADMM RECONSTRUCTIONS OF THE SL SINOGRAM SHOWN IN FIG.4. The SCORES ARE COMPUTEd INSIDE THE RECONSTRUCTION CIRCLE

\begin{tabular}{rrrr}
\hline & RMSE & PSNR & MSSIM \\
\hline ADMM-PSWF & 0.15 & 22.44 & 0.13 \\
\hline ADMM-KB & 0.15 & 22.47 & 0.13 \\
\hline ADMM-BSPLINE & 0.15 & 22.62 & 0.12 \\
\hline
\end{tabular}

This $\sigma$ fairly reproduces the noise level affecting the real data used in the following experiments. The reconstructed slices in Fig. 3, 4 show no difference at visual inspection and the metric scores (Tab. V, VI) confirm that the image quality is comparable for all SPS and ADMM implementations.

For the next two experiments, real data acquired at the TOMCAT beamline of the Swiss Light Source at the 


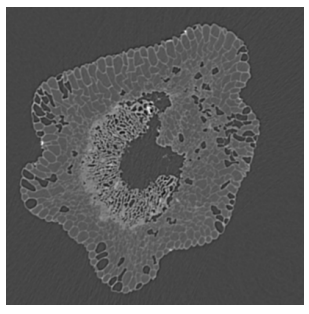

(a)

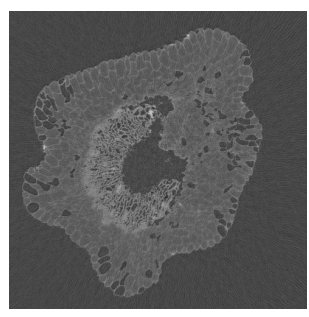

(b)

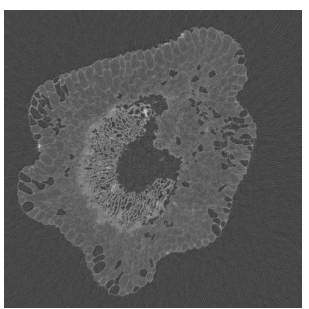

(c)

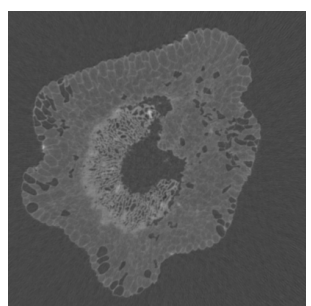

(d)

Fig. 6. ADMM reconstructions of the sinogram (300 views $\times 2560$ pixels) of a modern seed specimen. FBP-FULL is the FBP reconstruction of the fully sampled sinogram with 1441 views $\times 2560$ pixels Sample courtesy: S. Smith, University of Michigan. (a) Reconstr. with FBP-FULL. (b) Reconstr. with ADMM-PSWF. (c) Reconstr. with ADMM-KB. (d) Reconstr. with ADMM-BSPLINE.

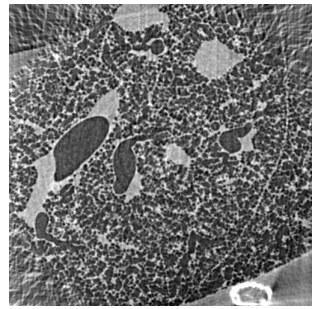

(a)

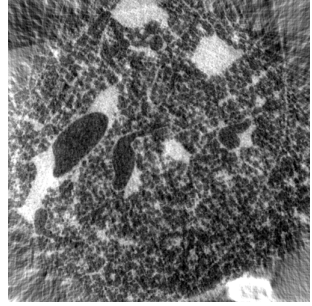

(b)

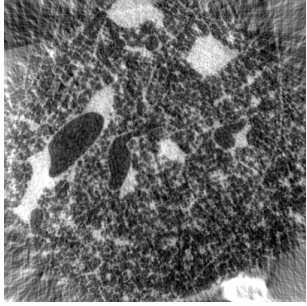

(c)

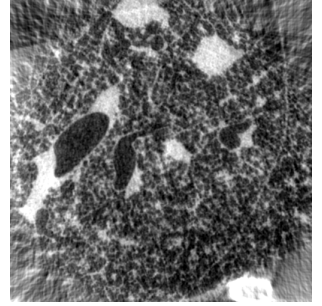

(d)

Fig. 7. SPS reconstructions of the sinogram (272 views $\times 2016$ pixels) of mouse lung tissue. FBP-FULL is the FBP reconstruction of the fully sampled sinogram with 901 views $\times 2016$ pixels. Sample courtesy: Lovric [59]. (a) Reconstr. with FBP-FULL. (b) Reconstr. with SPS-PSWF. (c) Reconstr. with SPS-KB. (d) Reconstr. with SPS-BSPLINE.

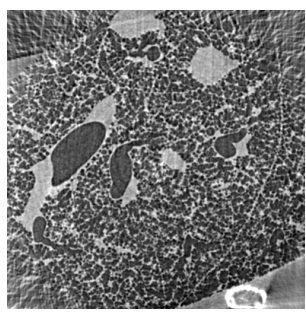

(a)

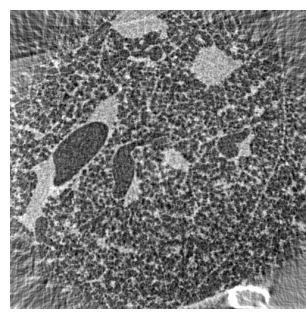

(b)

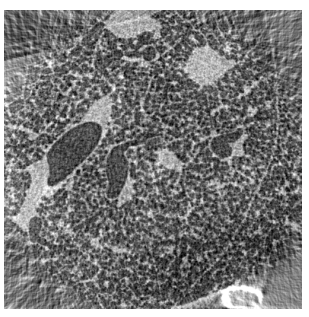

(c)

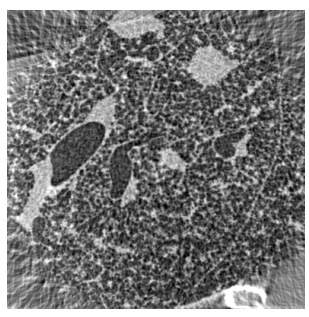

(d)

Fig. 8. ADMM reconstructions of the sinogram $(272$ views $\times 2016$ pixels $)$ of mouse lung tissue. FBP-FULL is the FBP reconstruction of the fully sampled sinogram with 901 views $\times 2016$ pixels. Sample courtesy: Lovric [59]. (a) Reconstr. with FBP-FULL. (b) Reconstr. with ADMM-PSWF. (c) Reconstr. with ADMM-KB. (d) Reconstr. with ADMM-BSPLINE.

TABLE VII

RMSE, PSNR AND MSSIM VALUES FOR THE SPS RECONSTRUCTIONS OF THE SinOGRAM OF THE MODERn SEED SPECIMEN SHOWN IN Fig.5. The SCORES ARE COMPUTEd INSIDE THE RECONSTRUCTION CIRCLE

\begin{tabular}{rrrr}
\hline & RMSE & PSNR & MSSIM \\
\hline SPS-PSWF & 0.06 & 31.33 & 0.14 \\
\hline SPS-KB & 0.06 & 31.33 & 0.14 \\
\hline SPS-BSPLINE & 0.07 & 30.56 & 0.10 \\
\hline
\end{tabular}

Paul Scherrer Institut have been used. In the first case, a sinogram with 1441 views $\times 2560$ pixels of a modern seed acquired in full tomography (i.e., the sample support is entirely in the FOV) has been downsampled to 300 views to create an underconstrained version of the original dataset. The effective detector pixel size is $2.9 \mu \mathrm{m}$.
TABLE VIII

RMSE, PSNR AND MSSIM VALUES FOR THE ADMM RECONSTRUCTIONS OF THE SINOGRAM OF THE Modern SeEd SPECIMEN SHown In Fig.6. The SCORES ARE COMPUTEd INSIDE THE RECONSTRUCTION CIRCLE

\begin{tabular}{rrrr}
\hline & RMSE & PSNR & MSSIM \\
\hline ADMM-PSWF & 8.10 & 29.96 & 0.38 \\
\hline ADMM-KB & 8.08 & 29.98 & 0.38 \\
\hline ADMM-BSPLINE & 7.83 & 30.25 & 0.13 \\
\hline
\end{tabular}

Fig. 5, 6 show the FBP reconstruction with Parzen filter of the original sinogram (FBP-FULL), followed by either the SPS (Fig.5) or the ADMM (Fig.6) reconstructions of the underconstrained dataset. FBP-FULL is used as reference image to compute the scores in Tab.VII, VIII. The iterative 
TABLE IX

RMSE, PSNR AND MSSIM VALUES FOR THE SPS RECONSTRUCTIONS OF The Sinogram of the Mouse Lung Tissue Shown in Fig.7. The SCORES ARE COMPUTED INSIDE THE RECONSTRUCTION CiRCLE

\begin{tabular}{rccr}
\hline & RMSE & PSNR & MSSIM \\
\hline SPS-PSWF & $4.23 \mathrm{e}-6$ & 18.44 & 0.48 \\
\hline SPS-KB & $4.23 \mathrm{e}-6$ & 18.44 & 0.48 \\
\hline SPS-BSPLINE & $4.87 \mathrm{e}-6$ & 17.21 & 0.35 \\
\hline
\end{tabular}

TABLE X

RMSE, PSNR AND MSSIM VALUES FOR THE ADMM RECONSTRUCTIONS OF THE SINOGRAM OF THE Mouse Lung Tissue ShOWN IN Fig.8. The SCORES ARE COMPUTED INSIDE THE RECONSTRUCTION CIRCLE

\begin{tabular}{rccr}
\hline & RMSE & PSNR & MSSIM \\
\hline ADMM-PSWF & $3.38 \mathrm{e}-6$ & 20.40 & 0.61 \\
\hline ADMM-KB & $3.47 \mathrm{e}-6$ & 20.14 & 0.60 \\
\hline ADMM-BSPLINE & $3.22 \mathrm{e}-6$ & 20.81 & 0.61 \\
\hline
\end{tabular}

reconstructions show again no evident differences at visual inspection except a slight blurring in the SPS-BSPLINE and ADMM-BSPLINE case. This image quality degradation is also reflected in the higher RMSE and lower MSSIM values for the implementations with RT-BSPLINE (Tab. VII, VIII).

In the second case, mouse lung tissue data acquired in interior tomography configuration (i.e. the sample support extends beyond the FOV) have been chosen. The original sinogram, consisting of 901 views $\times 2016$ pixels, has been downsampled to 272 views. The effective detector pixel size is $2.9 \mu \mathrm{m}$. Metric scores are computed against FBP-FULL as in the previous case. The SPS (Fig.7) and ADMM (Fig.8) reconstructions present no visible difference and the image quality assessed through the figures of merit (Tab. IX, X) lead to the same conclusion.

\section{Discussion AND CONCLUSION}

A novel application of the regridding method as forward projector with $O\left(N^{2} \log _{2} N\right)$ complexity for CT reconstruction has been presented. Forward regridding projectors (FRPs) with standard ( $\alpha=2.0$, FRP-PSWF) and minimal oversampling $(\alpha=1.125$, FRP-KB) have been studied.

The computational efficiency and accuracy of the proposed operators have been systematically compared with multiple state-of-the-art implementations of the Radon transform: a rayand distance-driven projector (RT-RAY, RT-DIST), a Radon transform based on a cubic B-spline basis (RT-BSPLINE), a fast hierarchical forward projector (FHFP) and a Radon transform based on the non-uniform fast Fourier transform with min-max interpolation (NUFFT). Among the algorithms with $O\left(N^{2} \log _{2} N\right)$ complexity, FRP-KB is on average faster by a factor of 4,11 and 21 with respect to FRP-PSWF, NUFFT and FHFP. Compared to pixel-based projectors like RT-BSPLINE, the speed increase achieved with FRP-KB is significantly larger (up to 3 orders of magnitude). Memory allocation for FRP-KB is reduced by $64 \%$ and $70 \%$ compared to the Fourier-based projectors FRP-PSWF and NUFFT. The accuracy of the FRP operators is slightly inferior compared to the other implementations of the Radon transform, although differences in the computed sinograms are barely visible upon visual inspection. We have shown that this lower accuracy becomes negligible when each sinogram is reconstructed with the related ramp-filtered backprojector, indicating that other aspects involved in the reconstruction process (e.g. filtering) have a stronger influence on the accuracy of the obtained results.

In a second step, the proposed forward regridding operators (FRP-PSWF and FRP-KB) have been integrated inside two different iterative schemes, (the separable paraboloidal surrogate, SPS, and the alternate direction method of multipliers, ADMM) to asses their performance for the reconstruction of simulated and real underconstrained tomographic datasets. These iterative algorithms have been benchmarked against the same iterative schemes implemented with RT-BSPLINE, the most accurate projector among those tested in this study. The results show that, despite the inferior accuracy of the fast operators based on the regridding method, both SPS/ ADMM-PSWF and SPS/ADMM-KB have a similar convergence rate and provide comparable image quality as SPS/ ADMM-BSPLINE. This result suggests that the minimization of the cost function in the tomographic reconstruction process is not strongly influenced by the chosen forward projector.

To conclude, we show that the proposed forward regridding projector with minimal oversampling (FRP-KB), thanks to its high computational efficiency, is an interesting new operator able to substantially speed up any iterative tomographic reconstruction algorithm, while preserving the results accuracy achieved with more sophisticated operators. Its low computational cost and reduced memory requirements make the proposed projector, so far developed for the parallel beam case, very appealing for addressing problems characterized by more complex geometries, usually very demanding especially from the memory point of view.

\section{APPENDIX \\ C-Like Pseudocode of THE FRP}

The following pseudocode refers to an implementation of FRP, where the convolution LUT is sampled with nearest neighbour scheme. The notation is the same as introduced in section II. In addition, $l=W / 2$ is the half width of the convolving kernel. The subscript $p$ stands for "padded". The parentheses $\lfloor\cdots\rfloor$ correspond to the floor operator, $\lceil\cdots\rceil$ to the ceil operator and $[\cdots]$ to the round operator. Although the pseudocode refers to our C implementation of the FRP, there are a couple of functions (the slicing operator "." and the memory allocating function "zeros"), that are characteristic of high-level programming languages like Matlab or Python, and are used to express some passages in a more concise form.

1) compute $\mathbf{I}_{p} \longleftarrow \operatorname{zeropad}(\mathbf{I})$ up to $G$ rows and columns

2) pre-removal of the interpolation kernel for $i=0, \ldots, G-1 \mathrm{do}$ : 
for $j=0, \ldots, G-1$ do:

$$
\underset{\text { endfor }}{\mathbf{I}_{p}^{(m)}[i, j] \longleftarrow} \longleftarrow \frac{\mathbf{I}_{p}[i, j]}{\psi\left[g_{2} i\right] \psi\left[g_{2} j\right]} ;
$$

endfor

3) compute $\hat{\mathbf{I}}_{p}^{(m)} \longleftarrow F F T 2\left(\mathbf{I}_{p}^{(m)}\right)$;

4) initialize $\hat{\mathbf{S}}_{p}=\operatorname{zeros}(M, G)$;

5) do interpolation in the Fourier space:

for $i=0, \ldots, M-1 \mathrm{do}$ :

for $k=0, \ldots, G / 2-1 \mathrm{do}$

$k_{x}=k \cos (\theta[i])+G / 2$

$k_{y}=k \sin (\theta[i])+G / 2$

$a_{k_{x}}=\left\lceil k_{x}-l\right\rceil, b_{k_{x}}=\left\lfloor k_{x}+l\right\rfloor$

$a_{k_{y}}=\left\lceil k_{y}-l\right\rceil, b_{k_{y}}=\left\lfloor k_{y}+l\right\rfloor$

for $k 1=a_{k_{x}}, \ldots, b_{k_{x}}$ do:

$\hat{\psi}_{x}=\hat{\psi}\left[g_{1} \cdot\left\lfloor\left|k_{x}-k_{1}\right| 7\right]\right.$

for $k 2=a_{k_{y}}, \ldots, b_{k_{y}}$ do:

$\hat{\boldsymbol{\psi}}_{y}=\hat{\boldsymbol{\psi}}\left[g_{1} \cdot\left\lfloor\left|k_{y}-k_{2}\right|\right\rceil\right]$

$\hat{\boldsymbol{\psi}}_{x, y}=\hat{\boldsymbol{\psi}}_{x} \cdot \hat{\boldsymbol{\psi}}_{y}$

$\hat{\mathbf{S}}_{p}[i, k]+=\hat{\mathbf{I}}_{p}^{(m)}[k 1, k 2] \cdot \hat{\boldsymbol{\psi}}_{x, y}$

$\hat{\mathbf{S}}_{p}[i, G-k]+=\hat{\mathbf{I}}_{p}^{(m)}[G-k 1, G-k 2] \cdot \hat{\boldsymbol{\psi}}_{x, y}$

end for on Cartesian index $k_{2}$

end for on Cartesian index $k_{1}$

$\hat{\mathbf{S}}_{p}[i, k]=\hat{\mathbf{S}}_{p}[i, k] \cdot e^{-\pi i k G}$

$\hat{\mathbf{S}}_{p}[i, G-k]=\hat{\mathbf{S}}_{p}[i, G-k] \cdot e^{\pi i k G}$

end for loop on radial variable $k$

$\mathbf{S}_{p}[i,:]=\operatorname{IFFT1}\left(\hat{\mathbf{S}}_{p}[i,:]\right)$

end for loop on angular index $i$

6) crop padded sinogram: $\mathbf{S}=\mathbf{S}_{p}[:, i 1: i 2]$

where $i 1=\lfloor(G-N) / 2\rceil \quad i 2=i 1+N$

7) return $\mathbf{S}$.

\section{REFERENCES}

[1] G. N. Ramachandran and A. V. Lakshminarayanan, "Three-dimensional reconstruction from radiographs and electron micrographs: Application of convolutions instead of Fourier transforms," Proc. Nat. Acad. Sci. USA, vol. 68, no. 9, pp. 2236-2240, 1971.

[2] G. T. Herman, Ed., Image Reconstruction From Projections: Implementation and Applications. Berlin, Germany: Springer-Verlag, 1979, pp. 81-104.

[3] A. C. Kak and M. Slaney, Principles of Computerized Tomographic Imaging. Piscataway, NJ, USA: IEEE Press, 1988.

[4] E. C. Frey, Z.-W. Ju, and B. M. W. Tsui, "A fast projector-backprojector pair modeling the asymmetric, spatially varying scatter response function for scatter compensation in SPECT imaging," IEEE Trans. Nucl. Sci. vol. 40, no. 4, pp. 1192-1197, Aug. 1993.

[5] G. L. Zeng, Y.-H. Hsieh, and G. T. Gullberg, "A rotating and warping projector/backprojector for fan-beam and cone-beam iterative algorithm," IEEE Trans. Nucl. Sci., vol. 41, no. 6, pp. 2807-2811, Dec. 1994.

[6] P. M. Joseph, "An improved algorithm for reprojecting rays through pixel images," IEEE Trans. Med. Imag., vol. 1, no. 3, pp. 192-198, Nov. 1982

[7] D.-C. Yu and S.-C. Huang, "Study of reprojection methods in terms of their resolution loss and sampling errors," IEEE Trans. Nucl. Sci., vol. 40, no. 4, pp. 1174-1178, Aug. 1993.

[8] P. A. Toft and J. A. Sørensen, "The radon transform-Theory and implementation," Ph.D. dissertation, Dept. Math. Model., Denmark Tech. Univ., Kongens Lyngby, Denmark, 1996.

[9] R. I. Siddon, "Fast calculation of the exact radiological path for a threedimensional CT array," Med. Phys., vol. 12, pp. 252-255, Mar. 1985.

[10] B. De Man and S. Basu, "Distance-driven projection and backprojection," in Proc. IEEE Nucl. Sci. Symp. Conf. Rec., vol. 3. Nov. 2002, pp. 1477-1480.
[11] L. M. Popescu and R. M. Lewitt, "Ray tracing through a grid of blobs," in Proc. IEEE Nucl. Sci. Symp. Conf. Rec., vol. 6. Oct. 2004, pp. 3983-3986.

[12] S. Horbelt, M. Liebling, and M. Unser, "Discretization of the radon transform and of its inverse by spline convolutions," IEEE Trans. Med. Imag., vol. 21, no. 4, pp. 363-376, Apr. 2002.

[13] W. J. Palenstijn, K. J. Batenburg, and J. Sijbers, "Performance improvements for iterative electron tomography reconstruction using graphics processing units (GPUs)," J. Struct. Biol., vol. 176, no. 2, pp. 250-253, 2011.

[14] C. R. Crawford, J. G. Colsher, N. J. Pelc, and A. H. R. Lonn, "High speed reprojection and its applications," Proc. SPIE, vol. 0914, pp. 311-318, Jun. 1988.

[15] M. L. Brady and W. Yong, "Fast parallel discrete approximation algorithms for the radon transform," in Proc. 4th Annu. ACM Symp. Parallel Algorithms Archit., Jul. 1992, pp. 91-99.

[16] M. L. Brady, "A fast discrete approximation algorithm for the radon transform," SIAM J. Comput., vol. 27, pp. 107-119, Feb. 1998.

[17] W. A. Gotz, "Ein schnelle diskrete radon transformation basierend auf rekursiv definierten digitalen geraden," Ph.D. dissertation, Univ. Innsbruck, Innsbruck, Austria, 1993.

[18] W. A. Götz and H. G. Druckmüller, "A fast digital radon transformAn efficient means for evaluating the Hough transform," Pattern Recognit., vol. 28, p. 711-718, 1996.

[19] P. E. Danielsson, "Iterative techniques for projection and backprojection," Dept. Elect. Eng., Linköping Univ., Linköping, Sweden, Tech. Rep. ISSN 1400-3902, Jun. 1997.

[20] A. Brandt and J. Dym, "Fast calculation of multiple line integrals," SIAM J. Sci. Comput., vol. 20, no. 4, pp. 1417-1429, 1999.

[21] A. Boag, Y. Bresler, and E. Michielssen, "A multilevel domain decomposition algorithm for fast $\mathrm{O}\left(\mathrm{N}^{2} \log \mathrm{N}\right)$ reprojection of tomographic images," IEEE Trans. Imag. Process., vol. 9, no. 9, pp. 1573-1582, Sep. 2000.

[22] S. Basu and Y. Bresler, "O $\left(\mathrm{N}^{2} \log _{2} \mathrm{~N}\right)$ filtered backprojection reconstruction algorithm for tomography," IEEE Trans. Imag. Process., vol. 9 , no. 10 , pp. $1760-1773$, Oct. 2000.

[23] T. Pipatsrisawat, A. Gacic, F. Franchetti, M. Püschel, and J. M. F. Moura, "Performance analysis of the filtered backprojection image reconstruction algorithms," in Proc. IEEE ICASSP, vol. 5. Mar. 2005, pp. 153-156.

[24] J. A. Fessler and B. P. Sutton, "Nonuniform fast Fourier transforms using min-max interpolation," IEEE Trans. Signal Process., vol. 51, no. 2, pp. 560-574, Feb. 2003.

[25] S. Matej, J. A. Fessler, and I. G. Kazantsev, "Iterative tomographic image reconstruction using Fourier-based forward and back-projectors," IEEE Trans. Med. Imag., vol. 23, no. 4, pp. 401-412, Apr. 2004.

[26] R. N. Bracewell and A. C. Riddle, "Inversion of fan-beam scans in radio astronomy," Astrophys. J., vol. 150, no. 2, pp. 427-434, 1967.

[27] W. N. Brouw, "Aperture synthesis," in Methods in Computational Physics, vol. 14. New York, NY, USA: Academic, 1975, pp. 131-175.

[28] H. Schomberg and J. Timmer, "The gridding method for image reconstruction by Fourier transformation," IEEE Trans. Med. Imag., vol. 14, no. 3, pp. 596-607, Sep. 1995.

[29] H. Sedarat and D. G. Nishimura, "On the optimality of the gridding reconstruction algorithm," IEEE Trans. Med. Imag., vol. 19, no. 4, pp. 306-317, Apr. 2000.

[30] J. D. O’Sullivan, "A fast sinc function gridding algorithm for Fourier inversion in computer tomography," IEEE Trans. Med. Imag., vol. 4, no. 4, pp. 200-207, Dec. 1985.

[31] J. L. Jackson, "Selection of a convolution function for Fourier inversion using gridding [computerised tomography application]," IEEE Trans. Med. Imag., vol. 10, no. 3, pp. 473-478, Sep. 1991.

[32] J. Walden, "Analysis of the direct Fourier method for computer tomography," IEEE Trans. Med. Imag., vol. 19, no. 3, pp. 211-222, Mar. 2000.

[33] P. J. Beatty, D. G. Nishimura, and J. M. Pauly, "Rapid gridding reconstruction with a minimal oversampling ratio," IEEE Trans. Med. Imag., vol. 24, no. 6, pp. 799-808, Jun. 2005.

[34] S. Matej and I. G. Kazantsev, "Fourier-based reconstruction for fully 3-D PET: Optimization of interpolation parameters," IEEE Trans. Med. Imag., vol. 25, no. 7, pp. 845-854, Jul. 2006.

[35] F. Marone and M. Stampanoni, "Regridding reconstruction algorithm for real-time tomographic imaging," J. Synchrotron Radiat., vol. 19, no. 6 , pp. 1029-1037, 2012. 
[36] S.-R. Zhao and H. Halling, "A new Fourier method for fan beam reconstruction," in Proc. IEEE Nucl. Sci. Symp. Med. Imag. Conf. Rec., vol. 2. Oct. 1995, pp. 1287-1291.

[37] Y. Z. O'Connor and J. A. Fessler, "Fourier-based forward and backprojectors in iterative fan-beam tomographic image reconstruction," IEEE Trans. Med. Imag., vol. 25, no. 5, pp. 582-589, May 2006.

[38] S. Schaller, T. Flohr, and P. Steffen, "An efficient Fourier method for 3-D radon inversion in exact cone-beam CT reconstruction," IEEE Trans. Med. Imag., vol. 17, no. 2, pp. 244-250, Apr. 1998.

[39] D. Slepian and H. O. Pollak, "Prolate spheroidal wave functions, Fourier analysis and uncertainty-I," Bell Syst. Tech. J., vol. 40, no. 1, pp. 43-63, 1961.

[40] H. J. Landau and H. O. Pollak, "Prolate spheroidal wave functions, Fourier analysis and uncertainty-II," Bell Syst. Tech. J., vol. 40, no. 1, p. $65-84,1961$.

[41] V. Rokhlin and H. Xiao, "Approximate formulae for certain prolate spheroidal wave functions valid for large values of both order and band-limit," Appl. Comput. Harmon. Anal., vol. 22, no. 1, pp. 105-123, 2007.

[42] J. F. Kaiser, "Non recursive digital filter design using the iosinh window function," in Proc. IEEE Int. Symp. Circuit Theory, 1974, pp. 20-23.

[43] L. A. Shepp and B. F. Logan, "The Fourier reconstruction of a head section," IEEE Trans. Nucl. Sci., vol. 21, no. 3, pp. 21-43, Jun. 1974

[44] M. Frigo and S. G. Johnson, "FFTW: An adaptive software architecture for the FFT," in Proc. Int. Conf. Acoust., Speech, Signal Process., vol. 3. May 1998, pp. 1381-1384.

[45] A. Dutt and V. Rokhlin, "Fast Fourier transforms for nonequispaced data," SIAM J. Sci. Comput., vol. 14, no. 6, pp. 1368-1393, 1993.

[46] G. Steidl, "A note on fast Fourier transforms for nonequispaced grids," Adv. Comput. Math., vol. 9, no. 3, pp. 337-352, 1998.

[47] N. Nguyen and Q. H. Liu, "The regular Fourier matrices and nonuniform fast Fourier transforms," SIAM J. Sci. Comput., vol. 21, no. 1, pp. 283-293, 1999.

[48] D. Potts, G. Steidl, and M. Tasche, "Fast Fourier transforms for nonequispaced data: A tutorial," in Modern Sampling Theory: Mathematics and Applications. Boston, MA, USA: Birkhäuser, 2001, pp. 247-270.

[49] Q. Huynh-Thu and M. Ghanbari, "Scope of validity of PSNR in image/video quality assessment," Electron. Lett., vol. 44, no. 13, pp. 800-801, Jun. 2008.

[50] Z. Wang, A. C. Bovik, H. R. Sheikh, and E. P. Simoncelli, "Image quality assessment: From error visibility to structural similarity," IEEE Trans. Imag. Process., vol. 13, no. 4, pp. 600-612, Apr. 2004.

[51] M. Lustig, D. Donoho, and J. M. Pauly, "Sparse MRI: The application of compressed sensing for rapid MR imaging," Magn. Reson. Med., vol. 58, no. 6, pp. 1182-1195, 2007.

[52] J. Fessler et al. Image Reconstruction Toolbox. [Online]. Available: http://web.eecs.umich.edu/ fessler/code/

[53] H. Erdogan and J. A. Fessler, "Monotonic algorithms for transmission tomography," IEEE Trans. Med. Imag., vol. 18, no. 9, pp. 801-814, Sep. 1999.

[54] K. Lange, "Convergence of EM image reconstruction algorithms with Gibbs smoothing," IEEE Trans. Med. Imag., vol. 9, no. 4, pp. 439-446, Dec. 1990.

[55] S. Boyd, N. Parikh, E. Chu, B. Peleato, and J. Eckstein, "Distributed optimization and statistical learning via the alternating direction method of multipliers," Found. Trends Mach. Learn., vol. 3, no. 1, pp. 1-122, Jan. 2011.

[56] L. I. Rudin, S. Osher, and E. Fatemi, "Nonlinear total variation based noise removal algorithms," Phys. D, Nonlinear Phenomena, vol. 60 , pp. 259-268, Nov. 1992.

[57] M. Nilchian, C. Vonesch, P. Modregger, M. Stampanoni, and M. Unser, "Fast iterative reconstruction of differential phase contrast X-ray tomograms," Opt. Exp., vol. 21, no. 5, pp. 5511-5528, 2013.

[58] J. A. Scales, "Tomographic inversion via the conjugate gradient method," Geophysics, vol. 52, no. 2, pp. 179-185, 1987.

[59] G. Lovric et al., "Dose optimization approach to fast X-ray microtomography of the lung alveoli," J. Appl. Cristallogr., vol. 46, pp. 856-860, Jun. 2013

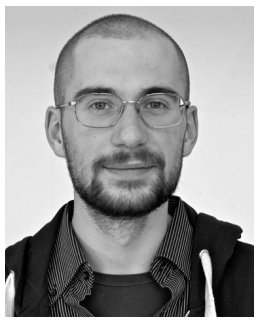

Filippo Arcadu received the B.Sc. and M.Sc. degrees from the Department of Physics and Astrophysics, University of Florence, in 2009 and 2012, respectively. He is currently pursuing the Ph.D. degree with the Institute for Biomedical Engineering, ETH, and the Xray Tomography Group of the Swiss Light Source, Paul Scherrer Institute. During his studies, he was a Software Developer with the Center for Protontherapy, Paul Scherrer Institute. His work focuses on the development of reconstruction algorithms for underconstrained tomographic datasets.

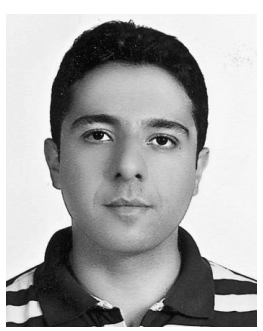

Masih Nilchian received the M.S. degree in electrical engineering and communication system from the Sharif University of Technology, Tehran, Iran, in 2008. He is currently pursuing the $\mathrm{Ph} . \mathrm{D}$. degree with the Biomedical Imaging Laboratory, École Polytechnique Fédérale de Lausann, Lausanne, Switzerland. He was involved in the applications of information and coding theory in biology and bioinformatics project with the Information System and Security Laboratory, Sharif University. $\mathrm{He}$ was the Designer of a genetic data bank with the Capability of Search for Genetic Center, Baghiyatallah University. From 2008 to 2010, he was a Research Officer of the MIMOS Laboratory with University Technology Malaysia. He has interest in the use of spline for image processing, information theory, coding theory, wavelet and multiresolution algorithms, and iterative methods for reconstruction. His main research area is in phase retrieving in differential phase contrast and phase contrast X-ray computed tomography.

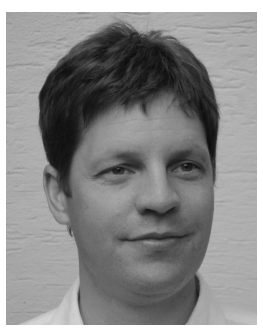

Alain Studer received the M.Sc. degree in physics from the University of Zürich, in 1998. From 2003 to 2006, he was working on the variability analysis of thalamic structures in the human brain with the Computer Vision Laboratory, Swiss Federal Institute of Technology. From 2006 to 2009, he was a member of the Mathematical Modeling Group with the Swiss Tropical Institute, implementing stochastic models supposed to forecast the patterns of malaria transmission. Since 2009, he has been with the Scientific Computing Group, Paul Scherrer Institute, mainly involved in parallelizing tomographic reconstruction code.

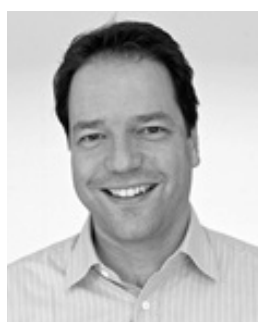

Marco Stampanoni received the Physics degree and the Ph.D. degree in physics from ETH Zürich, and the M.A.S. degree in medical physics from ETH. He has been an Associate Professor for X-ray imaging with the Institute for Biomedical Engineering, ETH, and the University of Zürich (since 2008) and the Head of the X-Ray Tomography Group of the Swiss Light Source with the Paul Scherrer Institute (since 2005). His work focuses on the development of novel X-ray imaging techniques based on synchrotron-radiation and laboratory sources.

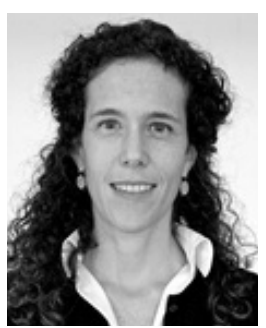

Federica Marone received the Earth Sciences degrees with a specialization in seismology and the $\mathrm{Ph} . \mathrm{D}$. degree in seismology dedicated to seismic tomography from ETH Zürich. She has been a Beamline Scientist with TOMCAT Beamline, Swiss Light Source, Paul Scherrer Institute, since 2006. Her current research focuses on the development of novel algorithms for tomographic reconstruction (analytic and iterative approaches), mitigation of artifacts, phase retrieval, and quantitative data analysis. 